\section{European \\ Neurology}

\title{
Message from the Editor
}

\author{
J. Bogousslavsky, Montreux
}

It is the policy of the journal to rotate the members of the Editorial Board every now and then and I would like to thank the following people for their great efforts over the past years:

D. Adams, Paris

A. Carolei, L’Aquila-Coppito

M. Chatel, Nice

H.H. Jung, Zurich

K. Roos, Indianapolis, Ind.

I would also like to welcome the new members of the Editorial Board and thank them for agreeing to support European Neurology:

G. Deuschl, Kiel

T. Karapanayiotides, Thessaloniki

S. Sacco, Aquila

L. Tatu, Besançon

A. Tsiskaridze, Tbilisi

P. Vuilleumier, Geneva
The following names are those of ad hoc reviewers, to whom I would also like to express my gratitude (January 2011-December 2011):

H.J. Abuhusain, Sydney, N.S.W.

H. Adams, Iowa City, Iowa

A.V. Alexandrov, Houston, Tex.

P. Allain, Angers

R. Alp, Tekirdag

M.U. Aluclu, Diyarbakir

S. Amaro, Barcelona

S. Amin-Hanjani, Chicago, Ill.

C. Angelini, Padova

J. Ankri, Paris

J.-M. Annoni, Fribourg

C.A. Antoniades, Oxford

C.T Antrion, Saint-Etienne

N. Araki, Saitama

A. Arboix, Barcelona

K. Arimura, Kagosjima

K. Asadullah, Berlin

F. Assal, Geneva

P. Aubourg, Paris

M.R. Azarpazhooh, Mashhad

H. Baezner, Stuttgart

P. Bain, London

T.H. Bak, Edinburgh

S. Bakchine, Reims

J. Barnes, London

F. Baronti, Tschugg

G. Barriere, Bordeaux

T. Bartsch, Kiel
H. Benamer, Birmingham S.R. Benbadis, Tampa, Fla. H. Benes, Schwerin

T. Bertorini, Memphis, Tenn.

E. Bezard, Bordeaux

D.C. Bezerra, Rio de Janeiro

J. Biller, Maywood, N.J.

B. Bjelke, Sigtuna

F. Blanc, Strasbourg

O. Boespflug Tanguy, Paris

K. Boetzel, Munich

J.-P. Brandel, Paris

B. Brochet, Bordeaux

J.M. Bronstein, Los Angeles, Calif.

E. Broussolle, Lyon-Bron

S. Brucki, São Paulo

E. Brusse, Rotterdam

J. Bufler, Wasserburg

J.-M. Burgunder, Bern

R. Businaro, Latina

P. Calabrese, Basel

P. Caliandro, Rome

L.R. Caplan, Boston, Mass.

S. Cappa, Milan

A. Carolei, L’Aquila-Coppito

A. Carota, Brissago

E. Carrera, Lausanne

V. Caso, Perugia

R.J. Castellani, Baltimore, Md.

Y. Celik, Edirne

H. Chabriat, Paris

A. Chakravarty, Calcutta

Y. Chang, Daegu

\section{KARGER}

Fax +41 613061234 E-Mail karger@karger.ch www.karger.com
(C) 2011 S. Karger AG, Basel

0014-3022/12/0671-0001\$38.00/0 
A.K. Chatterjee, Philadelphia, Pa.

A. Chaudhuri, Romford

C.-M. Chern, Taipei

C. Chin, San Francisco, Calif.

In Sup Choi, Burlington, Vt.

P.-W. Chung, Seoul

S. Chung, Sydney, N.S.W.

K.K. Chwojnicki, Gdansk

A. Conti, Messina

M. Cosottini, Pisa

J. Craig, Belfast

L. Crevits, Ghent

S. Cronin, Cork

A. Croquelois, Lausanne

F.A. de Falco, Naples

G. Defazio, Bari

G.R. de Freitas, Rio de Janeiro

M.R. de Freitas, Niterói

F. Deisenhammer, Innsbruck

M. Del Sette, La Spezia

J. de Reuck, Ghent

J. De Seze, Strasbourg

Vittorio Di Piero, Rome

D. Drachman, Worcester, Mass.

F. Ducray, Lyon

G.C. Ebers, Oxford

N.M.J. Edelstyn, Keele

M. El-Koussy, Bern

S.T. Engelter, Basel

T. Ettlin, Rheinfelden

A. Evoli, Rome

A. Felbecker, St. Gallen

A.A. Ferbert, Kassel

L. Ferini-Strambi, Milan

R. Ferri, Troina

J. Fiebach, Berlin

M. Fisher, Orange, Iowa

P. Flachenecker, Bad Wildbad

A. Fox, Toronto, Ont.

J.H. Friedman, Providence, Conn.

A. Frigeri, Bologna

T. Fukui, Yokohama

A. Gass, Basel

S. Gauthier, Montreal, Que.

A. Gerdelat-Mas, Toulouse

K.K. Ghandehari Mashhad, Razavi Khorasan

J.-A. Ghika, Sion

M.-F. Ghilardi, New York, N.Y.

I. Ghorayeb, Bordeaux

P. Giannakopoulos, Geneva

G. Giannakoulas, Thessaloniki

M.F. Giannoni, Rome

M. Giroud, Dijon

M. Gonzalez-Gay, Santander

R. Gottesman, Baltimore, Md.

C. Granziera, Lausanne

Harald Griese, Bad Oeynhausen

J.P. Guyot, Geneva

J. Haba-Rubio, Lausanne
G. Hadjigeorgiou, Larissa

G.F. Hamann, Wiesbaden

S. Happe, Bremen

H.-P. Hartung, Düsseldorf

R. Hashimoto, Nasushiobara

J.J. Hauw, Paris

M. Heath, London

C. Helmchen, Lübeck

J. Hendrikse, Utrecht

M.G. Hennerici, Mannheim

M.J. Hilz, Erlangen

M. Hirano, Sakai

B. Högl, Innsbruck

A.I. Holodny, New York, N.Y.

M. Hommel, Grenoble

H.B. Huttner, Erlangen

D. Inzitari, Florence

F. Ippolito, Rome

K. Ishihara, Tokyo

C. Jagella, Zurich

M.J. Jauss, Muehlhausen

J.-S. Jeng, Taipei

J.K. Jensen, Odense

A. Johansson, Stockholm

E. Juettler, Berlin

H.H. Jung, Zurich

G. Kaegi, St. Gallen

R.N. Kalaria, Newcastle upon Tyne

D.-W. Kang, Seoul

V. Kapetanakis, Cambridge

L J. Kappelle, Utrecht

T. Karapanayiotides, Thessaloniki

H. Karapinar, Sivas

S. Kaya, Trabzon

I. Kerkis, São Paulo

J. Kesselring, Valens

B.K. Kim, Seoul

J.S. Kim, Seoul

J.S. Kim, Seongnam-si

M.-W. Kim, Incheon

K. Kimura, Okayama

R. Kinno, Meguro-ku

H. Kinoshita, Toyonaka

A. Kirton, Calgary, Alta.

Y. Kitagawa, Tokyo

T. Kitazono, Fukuoka

M. Klein, Munich

U. Koedel, Munich

P.J. Koehler, Heerlen

R. Kollmar, Erlangen

J. Koziol, La Jolla, Calif.

E. Kumral, Izmir

A. Kunimatsu, Tokyo

S. Kusunoki, Osaka-Sayama

G. Kutlu, Ankara

S. Kuwabara, Chiba

S. Kuzuhara, Suzuka

P. Labauge, Nimes

P. Laloux, Yvoir

V. Lambrecq, Bordeaux
J. W. Langston, Sunnyvale, Calif.

V. Larrue, Toulouse

H. Lassmann, Vienna

B. Laurent, Saint-Etienne

M.S. Lee, Seoul

A. Leibovitz, Beer Yakov

F. Lenz, Baltimore, Md.

S. Leurgans, Chicago, Ill.

M. Levy Andersen, São Paulo

D. Leys, Lille

Li-M. Lien, Taipei

M. Linnebank, Zurich

X. Liu, Nanjing

E. Louis, New York, N.Y.

K.O. Lovblad, Geneva

C.G. Lund, Oslo

P. Maeder, Lausanne

A. Malafosse, Chêne-Bourg

M. Masserini, Monza

H. Matsuda Hidaka-city

F. Mattioli, Brescia

A. Maulaz, Porto Alegre

G. Mayer, Schwalmstadt-Treysa

J. McLeod, Camperdown, N.S.W.

S. Mead, London

R. Meeusen, Brussels

R. Mendez-Otero, Rio de Janeiro

B.L. Miller, San Francisco, Calif.

T. Misu, Sendai

H. Mitoma, Shinjyuku-ku

T. Miyamoto, Tochigi

F. Miyashita, Suita City

H. Mizusawa, Bunkyo-ku

M. Mlynash, Palo Alto, Calif.

J. Moncayo Gaete, Quito

T. Moreau, Dijon

G. Moris, Oviedo

N. Morris, Wolverhampton

T. Moulin, Besançon

E. Nagata, Isehara

H. Nakajima, Takatsukin

H. Naritomi, Osaka

B. Nater, Lausanne

M. Naumann, Augsburg

T. Neumann-Haefelin, Fulda

M. Niewada, Warsaw

P. Nilsson, Malmo

E. Nobile-Orazio, Milan

A. Novelli, Oviedo

J. Novy, Lausanne

D. Nyholm, Uppsala

S. Oberndorfer, Vienna

M. Oggioni, Siena

V.R. Oliveira, Lisboa

J.K. Olson, Madison, Wisc.

S.M. Oppenheimer, Baltimore, Md.

M. Paciaroni, Perugia

E. Palma, Rome

L. Pantoni, Florence

J.-H. Park, Goyang 
K.H. Park, Incheon

W. Paulus, Goettingen

A. Petzold, Amsterdam

B. Pfausler, Innsbruck

H.-W. Pfister, Munich

M. Pham, Heidelberg

F. Piehl, Stockholm

C. Pierrot-Deseilligny, Paris

S. Podnar, Ljubljana

A. Poggesi, Florence

J. Poirier, Paris

N. Poungvarin, Bangkok

S. Prabhakaran, Chicago, Ill.

F. Provini, Bologna

P. Pullicino, Buffalo, N.Y.

M. Radanovic, São Paulo

B. Rafal, Bangor

N.V. Ramani, Singapore

O. Rascol, Toulouse

S. Ricci, Perugia

A. Righini, Milan

G. Rizzo, Bologna

H. Rodgers, Newcastle upon Tyne

K. Roesler, Bern

O.M. Ronning, Lørenskog

A.O. Rossetti, Lausanne

E.J. Roth, Chicago, Ill.

M. Rousseaux, Lille

S. Roy, Lausanne

L. Rumbach, Besançon

D. Russell, Oslo

D. Ryglewicz, Warsaw

S. Sacco, L'Aquila

A. Saenz, San Sebastián

M.A. Samuels, Boston, Mass.

M. Sanborn, Philadelphia, Pa.

K. Schaller, Geneva

C. Schenk, Hennepin, Minn.

D. Schmidt, Berlin
R. Schmidt, Graz

A. Schnider, Geneva

M. Schoenberger, Freiburg

H. Schreiber, Ulm

M. Schrooten, Leuven

M. Schuepbach, Bern

S. Schwartz, Mannheim

S. Schwartzenberg, Rehovot

M. Seeck, Geneva

T. Seifert-Held, Graz

K. Seppi, Innsbruck

B. Sheng, Laichikok

J. Shiota, Yokohamashi

A. Shuaib, Edmonton, Alta.

C.J.M. Sindic, Brussels

I. Skoog, Göteborg

E. Smith, Calgary, Alta.

K. Sonka, Prague

J. Sorabjee, Mumbai

A.J. Steck, Basel

A. Straube, Munich

O. Suchowersky, Edmonton, Alta.

L. Suissa, Nice

S. Suskauer, Baltimore, Md.

S. Suzuki, Tokyo

B. Sweeney, Cork

K. Szabo, Mannheim

R. Sztajzel, Geneva

B. Tackenberg, Marburg

W.K. Tang, Hong Kong

L. Tatu, Besançon

A.H. Tayal, Pittsburgh, Pa.

B.E. Tettenborn, St. Gallen

T. Thomsen, Iowa City, Iowa

C.G. Thorpe, Exeter

N. Ticozzi, Milan

D. Toni, Rome

K. Toyoda, Suita

W. Tse, New York, N.Y.
A. Tsiskaridze, Tbilisi

T. Uchihara, Setagaya-ku

D. Uebelhart, Glion-sur-Montreux

K. Utsugisawa, Hanamaki

I. Uttner, Ulm

J. Valdueza, Bad Segeberg

J.M.L. Vallat, Limoges

N. van Alfen, Nijmegen

P. Van Damme, Leuven

P. Van den Bergh, Brussels

M. Viana-Baptista, Lisboa

E. Vicenzini, Rome

M. von Brevern, Berlin

P. Vuadens, Sion

H. Vuagnat, Bern

Y. Wakayama, Yokohama

C.W. Wallesch, Elzach

M. Wallin, Arlington, Tex.

O.W. Walusinski, Brou

D.J. Wang, Los Angeles, Calif.

B. Weder, St. Gallen

W.J. Weiner, Baltimore, Md.

D.J. Werring, London

C. Wider, Lausanne

J. Willeit, Innsbruck

J.B. Winer, Birmingham

M. Wintermark, Charlottesville, Va.

A.D. Wu, Los Angeles, Calif.

G. Xu, Nanjing

O.O. Yaldizli, Basel

M.K. Yamaguchi, Tokyo

H. Yamamoto, Tokyo

B.I. Yamout, Beirut

W.-K. Yoo, Chuncheon

H. Yoshikawa, Kanazawa

J. Zeng, Guangzhou

X. Zhu, Cleveland, Ohio 